\title{
CONTEXTO DE QUEDAS NOTIFICADAS EM UM HOSPITAL UNIVERSITÁRIO
}

\author{
CONTEXT OF FALLS NOTIFIED IN \\ A UNIVERSITY HOSPITAL
}

\section{EL CONTEXTO DE LAS CAÍDAS NOTIFICADAS EN UN HOSPITAL UNIVERSITARIO}

\author{
Sabrina Daros Tiensoli ${ }^{1}$ \\ Marina da Cruz Moreira ${ }^{2}$ \\ Sara Monteiro de Moraes ${ }^{3}$ \\ Fernanda Penido Matozinhos ${ }^{4}$ \\ Flávia Sampaio Latini Gomes ${ }^{4}$
}

Como citar este artigo: Tiensoli SD, Moreira MC, Morais SM, Matozinhos FP, Gomes FSL. Contexto de quedas notificadas em um hospital universitário. Rev baiana enferm. 2019;33:e32590.

Objetivo: analisar as circunstâncias das quedas notificadas em um hospital universitário de alta complexidade. Método: estudo descritivo, com abordagem quantitativa, realizado em um hospital universitário. A coleta de dados foi realizada de janeiro de 2015 a julho de 2016, por meio dos dados secundários, e em outubro de 2016. A mediana foi calculada para as variáveis contínuas com distribuição não normal e as frequências e proporções foram calculadas para as variáveis categóricas. Resultados: a taxa de incidência de quedas foi de 2,21\% ao mês. Considerando os dois momentos de coleta, respectivamente, as quedas foram mais frequentes na faixa-etária de 60 anos ou mais $(42,86 \%)$ e 20 a 59 anos (70,00\%), no turno da manhã (47,37\% e 60,00\%) e em pessoas com doenças cardiovasculares $(35,71 \%)$. Os profissionais que mais notificaram quedas foram enfermeiros. Conclusão: as circunstâncias das quedas notificadas em um hospital universitário de alta complexidade foram avaliadas na maioria dos casos e foram mais frequentes em pacientes do sexo masculino, com 60 anos ou mais, com doenças cardiovasculares, em uso de anti-hipertensivos e diuréticos. Além disso, notou-se que potencialmente existe subnotificação de quedas na instituição.

Descritores: Acidentes por Quedas. Segurança do Paciente. Enfermagem.

Objective: to analyze the circumstances of falls reported in a high-complexity university hospital. Method: a descriptive study with a quantitative approach, conducted in a university hospital. Data collection was performed from January 2015 to July 2016, using the secondary data, and in October 2016. The median was calculated for continuous variables with non-normal distribution and frequencies and proportions were calculated for categorical variables. Results: the incidence rate of falls was $2.21 \%$ per month. Considering the two collection moments, respectively, falls were more frequent in the age group 60 years old or older (42.86\%) and 20 to 59 years old (70.00\%), in the morning shift (47.37\% and 60.00\%) and in people with cardiovascular diseases (35.71\%). The professionals who most reported falls were nurses. Conclusion: the circumstances of falls reported in a bigh-complexity university hospital were assessed in most cases and were more frequent in male patients, aged 60 years old or older, with cardiovascular disease, using antihypertensive drugs and diuretics. In addition, it was noted that there is potentially underreporting of falls in the institution.

Descriptors: Accidents by Falls. Patient Safety. Nursing.

\footnotetext{
Enfermeira. Mestre em Enfermagem. Universidade Federal de Minas Gerais. Belo Horizonte, Minas Gerais, Brasil. sabrinadaros@hotmail.com Enfermeira. Residente multiprofissional em Enfermagem Obstétrica da Fundação Hospitalar do Estado de Minas Gerais. Belo Horizonte, Minas Gerais, Brasil. Médica. Mestre em Saúde do Adulto. Médica do Hospital das Clínicas da Universidade Federal de Minas Gerais. Belo Horizonte, Minas Gerais, Brasil.

Enfermeira. Doutora em Enfermagem. Professora da Universidade Federal de Minas Gerais. Belo Horizonte, Minas Gerais, Brasil.
} 
Objetivo: analizar las circunstancias de las caídas notificadas en un hospital universitario de alta complejidad. Método: estudio descriptivo con enfoque cuantitativo realizado en un hospital universitario. Los datos se recolectaron de enero de 2015 a julio de 2016, por medio de los datos secundarios, y en octubre de 2016. Se calculó la mediana para las variables continuas con distribución no normal, y se calcularon frecuencias y proporciones para las variables categóricas. Resultados: el indice de incidencia de caídas fue de 2,21\% por mes. Las caídas fueron más frecuentes en los grupos etarios de 60 años o más (42,86\%) y de 20 a 59 años (70,00\%), en el turno de la mañana (47,37\% y $60,00 \%)$ y en personas con enfermedades cardiovasculares $(35,71 \%)$. Los profesionales que notificaron más caídas fueron los enfermeros. Conclusión: las circunstancias de las caídas notificadas en un hospital universitario de alta complejidad se evaluaron en la mayoría de los casos, y fueron más frecuentes en pacientes de sexo masculino, de al menos 60 años, con enfermedades cardiovasculares, y en tratamiento con anti-hipertensivos y diuréticos. Además, se notó que existe un potencial de subnotificación de caídas en la institución.

Descriptores: Accidentes por Caídas. Seguridad del Paciente. Enfermería.

\section{Introdução}

As quedas podem ser definidas como um deslocamento não intencional do corpo para um nível inferior em relação a sua posição inicial, indo comumente ao chão, o que pode resultar em algum tipo de dano ${ }^{(1)}$. Estima-se que 424.000 quedas fatais ocorrem a cada ano mundialmente, fazendo delas a segunda maior causa de morte não intencional depois dos acidentes de trânsito ${ }^{(2)}$.

Este agravo pode decorrer de diversas causas, tais como: incapacidade cognitiva, uso de medicamentos, presença de algumas doenças, comprometimento sensorial e de funcionalidade, além das demográficas ${ }^{(1)}$. Deste modo, em qualquer faixa etária pode ser verificada a ocorrência de quedas, entretanto pessoas menores de cinco anos e as idosas são mais propensas a elas. Entre os idosos, a queda é uma das causas mais comuns de danos limitantes e deficiências, levando até mesmo à morte ${ }^{(3)}$.

Assim, na assistência à saúde, as quedas são consideradas eventos adversos (EA), isto é, incidentes que resultam em dano à saúde ${ }^{(1)}$, embora sejam erros preveníveis. Nos hospitais, a queda constitui uma das principais causas de eventos adversos, podendo chegar a 70\% de todas elas. Desses eventos intra-hospitalares, 30\% podem resultar em lesões físicas e 4 a 6\%, em lesões graves ${ }^{(4)}$.

No Brasil, em 2013, foi criado o Programa Nacional de Segurança do Paciente (PNSP), do qual é parte integrante o Protocolo de Prevenção de Quedas ${ }^{(1)}$. Este protocolo recomenda que o ambiente de cuidado seguro seja garantido, as estratégias de educação sejam promovidas, a queda deva ser notificada e o paciente avaliado e atendido imediatamente, para atenuação dos possíveis danos ${ }^{(1)}$.

A notificação dos casos, realizada pelos profissionais de saúde, permite a prestação do cuidado mais seguro, considerando que ela possibilita à equipe ter acesso às informações relacionadas à causa do evento, propor indicadores e adotar medidas de melhoria ${ }^{(1)}$.

Além disso, o PNSP prevê que as notificações sejam realizadas no Sistema de Informações em Vigilância Sanitária (Notivisa), de abrangência nacional, capaz de gerar dados necessários à publicação e divulgação periódica de boletins informativos, pela Agência Nacional de Vigilância Sanitária (Anvisa) ${ }^{(5)}$. A análise dos casos pode ser realizada de forma isolada ou agregada por essa Agência que, a seu critério, pode solicitar à instituição de saúde o detalhamento de determinado caso ${ }^{(5)}$.

Considerando tratar-se de uma prática recente nas instituições de saúde e de caráter não punitivo, nota-se que as notificações, ao longo dos anos, têm sido mais frequentes, mas ainda existem locais onde há subnotificação. Desses, o estado de Minas Gerais é o que possui atualmente o maior número de incidentes notificados, bem como o de Núcleos de Segurança do Paciente cadastrados. De março de 2014 a junho de 2019, em todo o Brasil, o Notivisa recebeu 
36.452 notificações de quedas de pacientes, sendo o segundo evento adverso mais notificado e o segundo mais relacionado a óbitos ${ }^{(6)}$.

Buscando compreender a notificação desse incidente em uma instituição hospitalar, este estudo teve como objetivo analisar as circunstâncias das quedas notificadas em um hospital universitário de alta complexidade.

\section{Método}

Trata-se de um estudo descritivo, com abordagem quantitativa de casos incidentes, realizado em um hospital universitário de grande porte da região Sudeste do Brasil. Além de ser considerado hospital de referência municipal e estadual de saúde no atendimento aos pacientes portadores de doenças de média e alta complexidade, nele são desenvolvidas atividades de ensino, pesquisa e extensão. Integrado ao Sistema Único de Saúde (SUS), atende uma clientela universalizada e exclusiva desse sistema ${ }^{(7)}$. As unidades funcionais abrangidas no estudo foram: Clínica Médica, Clínica Cirúrgica, Pediatria, Cuidado Intensivo Adulto - Centro de Tratamento e Terapia Intensiva (CTI) e Unidade Coronariana (UCO) -, Pronto-Socorro (PS) e Apoio Diagnóstico e Terapia por Imagem (ADTI).

A população do estudo foi constituída por todos os pacientes notificados por incidentes e EA registrados no sistema informatizado do hospital ( $\mathrm{n}=63$ notificações), sendo a amostra composta por 42 notificações, todas relacionadas ao evento queda. Foram incluídas todas as notificações de pacientes relacionadas a quedas, nas quais o campo "registro" estava preenchido. Foram excluídas 21 notificações que não continham o campo "registro" devidamente preenchido ou que, segundo a descrição do caso, não houve realmente a queda, de acordo com a definição da Organização Mundial de Saúde (OMS) ${ }^{(2)}$.

A coleta de dados deu-se em dois momentos distintos: o primeiro, de janeiro de 2015 a julho de 2016, por meio dos dados secundários das notificações de eventos adversos relacionados à queda, recebidas pelo Núcleo de Segurança do Paciente (NSP) da Instituição. Com base no número do registro do paciente, foi possível o acesso ao prontuário e a revisão dos registros, a fim de se obter informações sobre o contexto da queda.

As variáveis utilizadas no estudo foram: sexo, idade, circunstâncias da queda (tipo, turno, unidade funcional e local de ocorrência), causas, diagnósticos médicos primários e secundários, terapia endovenosa e medicamento em uso contínuo, avaliação quanto ao risco de queda, ocorrência de dano e consequência do dano, conduta da equipe após a queda, profissional notificador e registro da queda em prontuário. Nos casos em que o evento foi notificado por mais de um profissional, foi considerado apenas um evento e todos os profissionais que o notificaram.

O segundo momento de coleta de dados aconteceu visando identificar o número de quedas ocorridas no mês de outubro de 2016 na instituição do estudo. Os pesquisadores, diariamente, em busca ativa, coletavam informações sobre a ocorrência de quedas de pacientes em cada unidade com base no relato do enfermeiro responsável. Foi registrada, nesse mês, a ocorrência de 10 quedas. As variáveis utilizadas foram: sexo, faixa etária e circunstâncias da queda (tipo, turno, unidade funcional e local de ocorrência).

Neste estudo, foi calculada a taxa de incidência de quedas no período estudado. Os dados referentes à taxa de paciente-dia e número de internações foram fornecidos pelo Serviço de Arquivamento Médico e Estatístico (SAME) do hospital. Os dados foram analisados por meio do programa Stata, versão 14.0 (StataCorp, Texas, USA). A mediana foi calculada para as variáveis contínuas com distribuição não normal. Ademais, as frequências e proporções foram calculadas para as variáveis categóricas.

O estudo foi aprovado pelo Comitê de Ética em Pesquisa da Universidade Federal de Minas Gerais, sob Parecer n. 1.619.939.

\section{Resultados}

A taxa de incidência de quedas no período de janeiro de 2015 a julho de 2016 foi de 2,21 quedas ao mês. O índice geral de quedas, dos 42 pacientes notificados no período 
analisado, foi de 0,15 por 1.000 pacientes em todos os setores do hospital.

Dos 42 formulários analisados no estudo, observou-se que as notificações mais frequentes de quedas foram em pacientes do sexo masculino (54,76\%), na faixa etária de 60 anos ou mais (42,86\%), sendo a mediana das idades de 48,5 anos (IQ: 19-65). O turno de maior ocorrência de quedas foi o da manhã $(47,37 \%)$, nas unidades funcionais clínica médica e cirúrgica (ambas com 26,19\%). Em relação ao tipo de queda, a maioria foi da própria altura $(40,48 \%)$, seguida de berço/leito $(21,42 \%)$ e cadeira de banho $(14,29 \%)$. Os locais mais frequentes foram banheiro $(35,71 \%)$ e enfermaria/quarto $(33,33 \%)$ (Tabela 1). Deve-se esclarecer que o número total de pacientes pode não ser o mesmo para as variáveis estudadas, devido às diferentes taxas de respostas.

Tabela 1-Proporção de pacientes que sofreram quedas notificadas, segundo circunstâncias de ocorrências (tipo, turno, unidade funcional e local). Belo Horizonte, Minas Gerais, Brasil - 2015-2016 (n=42)

\begin{tabular}{|c|c|c|}
\hline Variáveis & $\mathbf{n}$ & $\%$ \\
\hline Tipo de queda & 42 & 100,00 \\
\hline Própria altura & 17 & 40,48 \\
\hline Leito/berço & 9 & 21,43 \\
\hline Cadeira de banho & 6 & 14,29 \\
\hline Maca & 5 & 11,90 \\
\hline Cadeira & 3 & 7,14 \\
\hline Vaso sanitário & 1 & 2,38 \\
\hline Cadeira de rodas & 1 & 2,38 \\
\hline Turno de Ocorrência da queda* & 38 & 100,00 \\
\hline Manhã & 18 & 47,36 \\
\hline Tarde & 10 & 26,32 \\
\hline Noite & 10 & 26,32 \\
\hline Unidade funcional & 42 & 100,00 \\
\hline Clínica médica & 11 & 26,19 \\
\hline Clínica cirúrgica & 11 & 26,19 \\
\hline Pediatria & 8 & 19,05 \\
\hline Pronto-socorro & 8 & 19,05 \\
\hline Apoio Diagnóstico e Terapia por Imagem & 3 & 7,14 \\
\hline Cuidados intensivos adulto & 1 & 2,38 \\
\hline Local de Ocorrência da Queda & 42 & 100,00 \\
\hline Banheiro & 15 & 35,72 \\
\hline Enfermaria/quarto & 14 & 33,33 \\
\hline Corredor & 8 & 19,05 \\
\hline Sala de exames & 4 & 9,52 \\
\hline Sala de emergência & 1 & 2,38 \\
\hline
\end{tabular}

Fonte: Elaboração própria.

* Os dados não estavam presentes em todos os formulários notificados por quedas.

Observou-se que a maioria das quedas notificadas foi causada por perda de equilíbrio (26,92\%), desorientação (17,31\%), falha/mau uso de equipamento $(17,31 \%)$ e fraqueza $(15,38 \%)$.

Quanto ao principal diagnóstico médico dos pacientes, considerados como primários, as doenças mais frequentes entre aqueles que caíram foram as do aparelho circulatório, representando 35,71\% e neoplasias, 23,81\%. Em relação aos diagnósticos secundários, a hipertensão arterial foi a comorbidade mais frequente 
entre os pacientes (26,56\%), seguida do diabetes e distúrbios neurológicos (ambos com 12,50\%).

A maior parte dos pacientes fazia uso de terapia endovenosa (78,57\%). Sobre os medicamentos em uso, os agentes anti-hipertensivos foram os mais utilizados (50,00\%), seguidos dos diuréticos $(32,14 \%)$ (Tabela 2).

Tabela 2-Proporção de quedas notificadas entre os pacientes, segundo diagnósticos médicos principais e secundários, terapias endovenosas e medicamentos de uso contínuo. Belo Horizonte, Minas Gerais, Brasil - 2015-2016 ( $n=42)$

\begin{tabular}{|c|c|c|}
\hline Variáveis & $\mathbf{n}$ & $\%$ \\
\hline Diagnósticos médicos primários* & 43 & 100,00 \\
\hline Doenças do aparelho cardiovascular & 15 & 34,88 \\
\hline Neoplasias & 10 & 23,80 \\
\hline Doenças do aparelho digestivo & 4 & 9,30 \\
\hline Doenças do aparelho respiratório & 3 & 6,98 \\
\hline $\begin{array}{l}\text { Outros (Síndrome da Imunodeficiência Humana Adquirida, sepse, } \\
\text { complicações de cuidados médicos) }\end{array}$ & 3 & 6,98 \\
\hline Doenças do sistema nervoso & 2 & 4,65 \\
\hline Doenças do aparelho urinário & 2 & 4,65 \\
\hline Doenças do aparelho osteomuscular & 2 & 4,65 \\
\hline Causas externas & 2 & 4,65 \\
\hline Diagnósticos secundários* & 64 & 100,00 \\
\hline Hipertensão arterial & 17 & 26,56 \\
\hline Diabetes mellitus & 8 & 12,50 \\
\hline Distúrbios neurológicos & 8 & 12,50 \\
\hline Doenças da tireoide & 7 & 10,94 \\
\hline Doença renal crônica & 7 & 10,94 \\
\hline Doenças cardiovasculares & 6 & 9,38 \\
\hline Doenças relacionadas à visão & 2 & 3,12 \\
\hline Doenças respiratórias & 2 & 3,12 \\
\hline Doenças autoimunes & 2 & 3,12 \\
\hline Neoplasias & 2 & 3,12 \\
\hline Outros (obesidade, síndrome de Down, amiloidose) & 3 & 4,69 \\
\hline Terapia endovenosa & 42 & 100,00 \\
\hline Sim & 33 & 78,57 \\
\hline Não & 9 & 21,43 \\
\hline Medicamento de uso contínuo** & 28 & 100,00 \\
\hline Anti-hipertensivos & 14 & 50,00 \\
\hline Diuréticos & 9 & 32,14 \\
\hline Tranquilizantes & 3 & 10,71 \\
\hline Narcóticos/opiáceos & 1 & 3,57 \\
\hline Antidepressivos tricíclico & 1 & 3,57 \\
\hline
\end{tabular}

Fonte: Elaboração própria.

* Um mesmo paciente pode apresentar mais de uma categoria da variável.

** Nem todos os pacientes faziam uso de medicamentos.

Sobre a avaliação do risco de queda, o notificador relatou que foi realizada pela equipe em 80,00\% dos casos. Observou-se que não houve diferença entre notificações com ou sem danos. Entretanto, dentre aquelas com danos, a maioria estava relacionada a danos 
não graves (73,68\%). Das 42 quedas notificadas, uma teve como consequência a morte do paciente. Após a ocorrência da queda, a conduta mais frequente foi a avaliação médica $(45,71 \%)$ e o paciente foi mantido em observação $(19,00 \%)$. O profissional que mais notificou as quedas foi o enfermeiro $(69,77 \%)$. Das quedas analisadas, 66,67\% foram registradas por algum profissional no prontuário do paciente (Tabela 3).

Tabela 3 - Proporção de quedas notificadas entre os pacientes, segundo avaliação quanto ao risco, ocorrência de dano, consequência do dano, conduta da equipe de saúde, profissional notificador e registro no prontuário. Belo Horizonte, Minas Gerais, Brasil - 2015-2016 (n=42)

\begin{tabular}{|c|c|c|}
\hline Variáveis & $\mathbf{n}$ & $\%$ \\
\hline Avaliação quanto ao risco de queda & 30 & 100,00 \\
\hline Sim & 24 & 80,00 \\
\hline Não & 6 & 20,00 \\
\hline Ocorrência de Dano & 38 & 100,00 \\
\hline Sim & 19 & 50,00 \\
\hline Não & 19 & 50,00 \\
\hline Consequência do Dano & 19 & 100,00 \\
\hline Não grave & 14 & 73,68 \\
\hline Causou incapacidade temporária & 4 & 21,05 \\
\hline Óbito & 1 & 5,26 \\
\hline Conduta da equipe de saúde após a queda* & 70 & 100,00 \\
\hline Avaliação médica & 32 & 45,71 \\
\hline Paciente mantido em observação & 19 & 27,14 \\
\hline Realizado exame de imagem & 11 & 15,71 \\
\hline Avaliação do enfermeiro & 7 & 10,00 \\
\hline Realizado eletroneuromiografia & 1 & 1,43 \\
\hline Profissional notificador* & 43 & 100,00 \\
\hline Enfermeiro & 30 & 69,77 \\
\hline Técnico de Enfermagem & 4 & 9,30 \\
\hline Médico & 3 & 6,98 \\
\hline Fisioterapeuta & 1 & 2,33 \\
\hline Outros** & 5 & 11,63 \\
\hline Registro da Queda no Prontuário & 42 & 100,00 \\
\hline $\operatorname{Sim}$ & 28 & 66,67 \\
\hline Não & 14 & 33,33 \\
\hline
\end{tabular}

Fonte: Elaboração própria.

* Um mesmo paciente pode apresentar mais de uma categoria da variável.

** Incluem: farmacêutico, estudante, técnico em radiologia, residente multiprofissional e residente médico.

Como resultado da segunda parte do estudo, foram identificadas 10 quedas por meio de busca ativa em prontuários. Não houve diferença entre sexo masculino e feminino (ambos com 50,00\%) e o evento foi mais frequente na faixa etária entre 20-59 anos, com 70,00\% das notificações.
Em relação ao tipo de queda, a maioria foi da própria altura $(90,00 \%)$. O turno de maior ocorrência de quedas foi o da manhã (60,00\%), nas unidades funcionais clínica médica $(50,00 \%)$ e pronto-socorro $(30,00 \%)$, e os locais mais frequentes foram enfermaria (50,00\%) e banheiro (40,00\%) (Tabela 4). 
Tabela 4 - Proporção de pacientes que sofreram quedas notificadas, segundo circunstâncias de ocorrências (tipo, turno, unidade funcional e local). Belo Horizonte, Minas Gerais, Brasil - out. 2016 $(\mathrm{n}=10)$

\begin{tabular}{l|c|c}
\hline Variáveis & $\mathbf{n}$ & $\mathbf{\%}$ \\
\hline Tipo de queda & $\mathbf{1 0}$ & $\mathbf{1 0 0 , 0 0}$ \\
Própria altura & 9 & 90,00 \\
Cadeira & 1 & 10,00 \\
Turno de ocorrência da queda & $\mathbf{1 0}$ & $\mathbf{1 0 0 , 0 0}$ \\
Manhã & 6 & 60,00 \\
Noite & 3 & 30,00 \\
Tarde & 1 & 10,00 \\
Unidade funcional & $\mathbf{1 0}$ & $\mathbf{1 0 0 , 0 0}$ \\
Clínica Médica & 5 & 50,00 \\
Pronto-Socorro & 3 & 30,00 \\
Clínica Cirúrgica & 2 & 20,00 \\
Local de ocorrência da queda & $\mathbf{1 0}$ & $\mathbf{1 0 0 , 0 0}$ \\
Enfermaria & 5 & 50,00 \\
Banheiro & 4 & 40,00 \\
Corredor & 1 & 10,00 \\
\hline
\end{tabular}

Fonte: Elaboração própria.

\section{Discussão}

As quedas fazem parte dos principais eventos adversos preveníveis em instituições de saúde ${ }^{(6)}$. Em 2019, em relatório publicado pela ANVISA, sobre os eventos adversos relacionados à assistência em saúde, notificados por 4.549 Núcleos de Segurança do Paciente no Brasil, as quedas constituíram o segundo evento adverso mais notificado no período de março de 2014 a junho de $2019^{(6)}$.

Em outro hospital universitário, no sul do Brasil, a incidência de quedas entre pacientes adultos nos anos de 2011 a 2015 foram, respectivamente, 1,61 quedas/1.000 pacientes; 2,03 quedas/1.000 pacientes; 1,83 quedas/1.000 pacientes; 1,62 e 1,42 quedas/1.000 pacientes $^{(8-9)}$. Neste estudo, os resultados mostraram que a incidência de quedas notificadas no período de 2015 a 2016 foi de 0,15 quedas/1.000 pacientes.

A menor ocorrência de quedas pode ser um indicativo de qualidade assistencial ${ }^{(1)}$. Entretanto, a incidência de quedas encontrada neste estudo pode sugerir subnotificação desse evento adverso na instituição. Tal fato pode ser reforçado quando se compara a ocorrência de quedas notificadas em 18 meses com aquelas que foram informadas pelos profissionais durante busca ativa por um mês (3,32 ao mês). A subnotificação nem sempre é intencional, mas pode estar relacionada ao desconhecimento, por parte dos profissionais, da importância da notificação dos EA, bem como ao medo diante de uma cultura punitiva nas instituições, prejudicando o processo de melhoria ${ }^{(5)}$.

Em relação às características dos pacientes que sofreram quedas notificadas, observou-se que a maioria era do sexo masculino e com idade igual ou superior a 60 anos. Sobre o sexo, estudos apresentaram resultados semelhantes e apontaram que o fato de ocorrer mais quedas em homens pode estar relacionado a fatores culturais, como maior dificuldade de homens em pedir e aceitar ajuda na execução de suas atividades diárias ${ }^{(10-11)}$.

Sobre a faixa etária, sabe-se que o avanço da idade desencadeia uma série de alterações fisiológicas, consideradas fatores de risco para a ocorrência de quedas, mais frequentes nessa 
faixa etária ${ }^{(1,9,11)}$. Os fatores comumente observados são aqueles relativos à instabilidade postural, alteração da marcha, incapacidade funcional e polifarmácia ${ }^{(9)}$.

Para compreender as notificações de queda no local do estudo (um hospital universitário de grande porte), foi importante analisar o contexto no qual o evento ocorreu. O turno de maior ocorrência de quedas notificadas foi o período da manhã, o que difere de outros estudos que apontam o período noturno como o mais frequente $^{(9,11)}$. Ressalta-se que o período da manhã pode oferecer riscos, pois é o momento do dia em que mais são realizados procedimentos e cuidados em saúde com os pacientes, tais como: banho, medicações, exames e estímulo, sempre que possível, estímulos à autonomia do paciente ao desenvolver suas atividades. No caso de um hospital escola, como o do estudo, no período da manhã também se concentra o maior número de estudantes em ensino clínico, aumentando as atividades e procedimentos realizados com os pacientes e, por conseguinte, as suas transferências entre leito, cadeira, maca e outros.

Em relação ao local da queda notificada, houve maior ocorrência nas clínicas médica e cirúrgica. Essas duas unidades correspondem, na instituição, à maioria dos leitos disponíveis nos setores de internação e recebem pacientes de diversas especialidades, tais como cardiologia, neurologia, transplante, oncologia e ortopedia. A maior ocorrência de quedas em unidades de internação clínica e cirúrgica pode ser justificada por longo tempo de permanência do paciente, idade avançada e maior complexidade da assistência $^{(6,8,11)}$.

Apesar das unidades de terapia intensiva serem os setores mais críticos do hospital, com pacientes em estado grave de saúde, também são os setores com maior vigilância por parte dos profissionais $^{(11)}$. Além disso, o paciente tende a ter maior restrição, principalmente em relação à sua mobilidade e deambulação, permanecendo no leito, protegido por grades.

Neste estudo, a maioria das quedas notificadas foi a da própria altura e ocorreu no banheiro e na enfermaria/quarto. A queda da própria altura foi o tipo de queda mais frequente em outros estudos ${ }^{(9,12)}$ e pode estar relacionada aos fatores intrínsecos do paciente, tais como desorientação, incapacidade cognitiva, fraqueza, uso de medicamentos e comorbidades ${ }^{(13)}$. Vale lembrar que não foi relatada a presença de obstáculos ou piso escorregadio nas notificações das quedas no período estudado, o que pode ser um indicativo de que a queda da própria altura estava relacionada aos fatores intrínsecos do paciente.

Quanto aos locais, a enfermaria/quarto e o banheiro são os mais utilizados pelos pacientes durante o período de internação hospitalar e, portanto, é comum que sejam apontados como os de maior ocorrência desse tipo de evento adverso $^{(6,9,11)}$. Das 36.452 notificações de quedas ocorridas em todo o país e recebidas pela Anvisa, no período de março de 2014 a junho de 2019, 21.296 (58\%) delas ocorreram no quarto e no banheiro ${ }^{(6)}$.

Outra variável importante para compreender o contexto das quedas notificadas foi o uso contínuo de medicamentos. Os medicamentos mais frequentemente utilizados pelos pacientes cujas notificações compuseram a amostra foram anti-hipertensivos e diuréticos, concordando com outros autores ${ }^{(1,9,14)}$. Os agentes hipertensivos e os diuréticos são drogas frequentemente utilizadas no tratamento de hipertensão arterial e em casos de doenças cardiovasculares. Seus efeitos são propícios à ocorrência de quedas, devido ao alto risco de hipotensão ortostática ou até mesmo diminuição do fluxo sanguíneo cerebral, com perda de consciência ${ }^{(9)}$. Os diuréticos ainda estão relacionados à urgência urinária, demandando agilidade do paciente para o acesso ao banheiro e podendo aumentar seu risco de cair.

Salienta-se que as doenças cardiovasculares, neste estudo, foram o diagnóstico mais frequente dos pacientes que sofreram quedas, assim como a hipertensão arterial foi a comorbidade mais comum entre eles, o que se relaciona com os medicamentos citados.

Outras classes de medicamentos associadas ao risco de quedas são aquelas que agem no sistema nervoso central, como os antidepressivos tricíclicos, tranquilizantes e os narcóticos/opiáceos ${ }^{(15)}$. No presente estudo, poucos pacientes estavam 
em uso dessas classes de medicamentos no período em que sofreram a queda.

O diabetes foi a segunda comorbidade mais frequente entre os pacientes que tiveram queda notificada. O uso de medicação hipoglicemiante e as complicações da doença, como neuropatia diabética e poliúria, podem ser considerados fatores que aumentam o risco de quedas, pois podem levar o paciente ao desmaio e causar instabilidade de marcha ${ }^{(1,16)}$.

Os fatores extrínsecos observados neste estudo como causa de queda são aqueles relacionados ao uso de equipamentos/dispositivos que auxiliam na marcha. Considerando-se que os fatores extrínsecos são modificáveis, é de fundamental importância que haja descrição completa, por parte dos notificadores, dos problemas apresentados pelo equipamento ou sua relação com o evento adverso. Dessa forma, a equipe do NSP poderá planejar e implementar medidas oportunas de prevenção para esse tipo de ocorrência.

Neste trabalho, dentre os pacientes que sofreram algum tipo de dano decorrente da queda, aqueles notificados como não graves, isto é, que não provocaram ou prolongaram o tempo de internação, foram os mais frequentes. Salienta-se que as quedas levam à ocorrência de danos graves em $20 \%$ a $30 \%$ dos $\operatorname{casos}^{(13)}$. Neste estudo, ocorreram cinco danos graves; destes, um $(5,26 \%)$ foi fatal.

O enfermeiro foi o profissional que mais notificou as quedas no período estudado. A equipe de enfermagem tem maior proximidade com o paciente e, portanto, mais chance de presenciar quedas. Além disso, podem ser esses os profissionais mais frequentemente solicitados para prestar ajuda na ocorrência do evento. Contudo, observou-se, neste estudo, que, em comparação com o médico, o enfermeiro não foi o profissional quem mais avaliou o paciente imediatamente após a queda $(45,7 \%$ e 10,0\% dos casos notificados, respectivamente) ou não fez o registro da avaliação, se realizada.

Por fim, diante de diversas situações que propiciam a ocorrência de quedas em pacientes hospitalizados, torna-se fundamental a avaliação e identificação dos riscos que envolvem o paciente para promover a sua prevenção. Neste estudo, 80,0\% dos pacientes foram avaliados quanto ao risco de queda, porém, as ações desenvolvidas pelos setores envolvidos não se mostraram eficazes a ponto de mitigar o evento. Uma das alternativas para se realizar essa avaliação de forma mais efetiva é por meio da Escala de Morse, que tem se mostrado eficaz na identificação do risco de queda de pacientes ${ }^{(14)}$.

O número de notificações analisadas foi um fator limitante deste estudo. Este fato ocorreu devido à subnotificação que provavelmente ocorre na instituição, previamente mencionada, bem como às perdas decorrentes de preenchimento incompleto ou incorreto dos formulários de notificação. São necessárias, portanto, ações de educação continuada que auxiliem os profissionais na identificação dos riscos de queda, suas principais formas de prevenção e notificação adequada de todos os eventos, contribuindo, assim, para a redução dos índices de quedas, aumento da segurança do paciente e melhoria da qualidade da assistência nos serviços de saúde.

\section{Conclusão}

As quedas foram mais frequentes em pacientes do sexo masculino, com 60 anos ou mais, com doenças cardiovasculares, em uso de anti-hipertensivos e diuréticos. Os profissionais que mais notificaram quedas foram enfermeiros e não houve diferença entre pacientes que sofreram dano ou não.

Observou-se que a maioria dos pacientes foi avaliada quanto ao risco de queda, porém as ações desenvolvidas não se mostraram plenamente eficazes. Além disso, notou-se que potencialmente existe subnotificação de quedas na instituição. Faz-se necessário, portanto, o estabelecimento de estratégias que garantam as notificações de eventos adversos de forma perene.

\section{Colaborações:}

1 - concepção, projeto, análise e interpretação dos dados: Sabrina Daros Tiensoli, Marina da Cruz Moreira, Sara Monteiro de Morais e Flávia Sampaio Latini Gomes; 
2 - redação do artigo e revisão crítica relevante do conteúdo intelectual: Sabrina Daros Tiensoli, Fernanda Penido Matozinhos e Flávia Sampaio Latini Gomes;

3 - aprovação final da versão a ser publicada: Sabrina Daros Tiensoli e Flávia Sampaio Latini Gomes.

\section{Referências}

1. Brasil. Ministério da Saúde. Agência Nacional de Vigilância Sanitária. Fundação Oswaldo Cruz. Protocolo prevenção de quedas. Brasília, DF; 2013.

2. World Health Organization. Falls: Fact Sheet [Internet]. Genebra; 2012 [cited 2019 Oct 2]. Available from: http://www.who.int/mediacentre/ factsheets/fs344/en/

3. Oliveira DU, Ercole FF, Melo LS, Matos SS, Campos CC, Fonseca EAM. Avaliação de quedas em idosos hospitalizados. Rev enferm UFPE on line. 2017;11(Supl. 11):4589-97. DOI: 10.5205/ reuol.11138-99362-1-SM.1111sup201707

4. Anderson DC, Postler TS, Dam TT. Epidemiology of hospital system patient falls: a retrospective analysis. Am J Med Qual. 2015;31(5):423-8. DOI: $10.1177 / 1062860615581199$

5. Brasil. Agência Nacional de Vigilância Sanitária. Gestão de riscos e investigação de eventos adversos relacionados à assistência à saúde. Brasília, DF; 2017.

6. Brasil. Agência Nacional de Vigilância Sanitária. Relatórios dos Estados - eventos adversos [Internet]. 2019 [cited 2019 Oct 7]. Available from: https://www20.anvisa.gov.br/ segurancadopaciente/index.php/publicacoes/ category/relatorios-dos-estados

7. Universidade Federal de Minas Gerais. Hospital das Clínicas da UFMG: institucional [Internet]. Belo Horizonte; 2019 [cited 2019 Oct 7]. Available from: http://www2.ebserh.gov.br/web/hc-ufmg/

8. Luzia MF, Cassola TP, Suzuki LM, Dias VLM, Pinho LB, Lucena AF. Incidence of falls and preventive actions in a University Hospital. Rev Esc Enferm USP. 2018;52:e03308. DOI: 10.1590/ s1980-220x2017024203308
9. Barbosa AS, Chaves EHB, Ribeiro RG, Quadros DV, Suzuki LM, Magalhães AMM. Caracterização dos incidentes de quedas de pacientes adultos internados em um hospital universitário. Rev Gaúcha Enferm. 2019;40(esp):e20180303. DOI: https://doi.org/10.1590/1983-1447.2019.20180303

10. Sousa KAS. Quedas de pacientes em um Hospital Público de Ensino [dissertação]. Belo Horizonte: Escola de Enfermagem, Universidade Federal de Minas Gerais; 2014.

11. Caveião C, Sales WB, Montezeli JH, Sena EM, Loureiro GM, Avanci MATM. Perfil clínico e consequências decorrentes de quedas em hospital universitário no Sul do Brasil. Rev Saúde Desenv. 2018 [cited 2019 Oct 7];12(10):183-96. Available from: https://www.uninter.com/revistasaude/index. php/saudeDesenvolvimento/article/view/877/506

12. Nomura ATG, Graeff MF, Pruinelli L, Almeida MA. Incidence of falls in a general hospital in Southern Brazil. Stud Health Technol Inform. 2019;264:1741-2. DOI: 10.3233/SHTI190625

13. Luzia MF, Victor MAG, Lucena AF. Diagnóstico de enfermagem risco de quedas: prevalência e perfil clínico de pacientes hospitalizados. Rev Latino-Am Enfermagem. 2014;22(2):262-8. DOI: 10.1590/0104-1169.3250.2411

14. Urbanetto JS, Creutzberg M, Franz F, Ojeda BS, Gustavo AS, Bittencourt HR, et al. Morse Fall Scale: tradução e adaptação transcultural para a língua portuguesa. Rev Esc Enferm USP. 2013;47(3). DOI: 10.1590/S0080-623420130000300007

15. Ribeiro TB, Melo DO, Maia FOM, Ribeiro E. Medication-related inpatient falls: a critical review. Braz J Pharm Sci. 2018; 54(1):e17355. DOI: 10.1590/ s2175-97902018000117355

16. Najafpour Z, Godarzi Z, Arab M, Yaseri M. Risk factors for falls in hospital in-patients: a prospective nested case control study. Int J Health Policy Manag. 2019;8(5):300-6. DOI: 10.15171/ ijhpm.2019.11

Recebido: 29 de julho de 2019 Aprovado: 17 de outubro de 2019 Publicado: 16 de março de 2020 
A Revista Baiana de Enfermagem utiliza a Licença Creative Commons - Atribuição-NãoComercial 4.0 Internacional. https://creativecommons.org/licenses/by-nc/4.0/

Este artigo é de acesso aberto distribuído sob os termos da Licença Creative Commons (CC BY-NC).

Esta licença permite que outros remixem, adaptem e criem a partir do seu trabalho para fins não comerciais. Embora os novos trabalhos tenham de lhe atribuir o devido crédito e não possam ser usados para fins comerciais, os usuários não têm de licenciar esses trabalhos derivados sob os mesmos termos. 